\title{
Optimizing Real-Time Wellness Intervention Studies in Healthcare Shift Workers: Outcomes from a Prospective, Single-subject Design Study of a Digital Sleep Aid
}

\author{
Nicole M. Duggan, MD \\ Brigham and Women's Hospital \\ Nmduggan@partners.org
}

Andrew J. Goldsmith, MD, MBA

Brigham and Women's Hospital Ajgoldsmith@partners.org

\author{
M. Adrian Hasdianda, MD, MSc \\ Brigham and Women's Hospital \\ Mhasdianda@bwh.harvard.edu
}

Anna Condella, MD

University of Washington Alc127@uw.edu
Guru Jambaulikar, MBBS, MPH

Brigham and Women's Hospital

Gjambaulikar@bwh.harvard.edu

Adaira I. Landry, MD, MEd

Brigham and Women's Hospital Ailandry@bwh.harvard.edu
Edward W. Boyer, MD, PhD

Ohio State University

eboyer@bwh.harvard.edu
Andrew J. Eyre

Brigham and Women's Hospital $\underline{\text { Aeyre@ } @ \text { bwh.harvard.edu }}$

\begin{abstract}
Sleep disorders associated with shift work impair alertness and increase risk of chronic physical and mental health disease. Though studying sleep aids in atrisk populations such as shift workers is critical to improving wellness, implementing robust studies to evaluate wellness interventions in live populations can be particularly challenging. We performed a prospective, single-subject design study in which we assessed the effect of noise-masking earbuds on sleep quality, sleepiness, and stress level in health care shift workers. Despite being gifted the technology at the end of the study, we faced poor participant accrual and study retention. Additionally, robust analysis of our intervention's impact on post-shift alertness was underpowered due to variable participant scheduling. In order to make meaningful advances in improving wellness among such groups, studies emphasizing objective outcome measures while minimizing participant obligation to study protocols are ideal. Here, we discuss our experience studying a digital sleep intervention in a real-time setting and propose solutions for improving subject accrual, retention and methods for objective data collection in studies performed in a live shift worker population.
\end{abstract}

\section{Introduction}

It is estimated that one-third of adults in the United States currently do not get the recommended seven hours of sleep nightly, and at least 50 million Americans suffer from chronic sleep disorders.[1,2] Shift workers often engage in overnight working hours, variable or rotating schedules, or extended hours on-call hours all of which contribute to uniquely worse sleep.[3-6] Healthcare work is an occupation with some of the highest prevalence of sleep disturbances (e.g. multiple awakenings), and sleep deficiency due to either extended wake episodes (i.e., acute sleep deprivation), multiple days/nights of insufficient sleep (i.e., sleep restriction or chronic sleep deprivation), and emotional and psychological challenges associated with the work.[7]

In healthcare workers sleep loss impairs alertness, which can lead to poor work performance and medical errors potentially compromising patient care.[4,8-11] Despite the recognized impact of sleep loss on clinician health and patient safety, when compared to other wellness initiative, workplace interventions to combat sleep loss and improve restful sleep are lacking.[12] Studying live sleep interventions in healthcare workers can be challenging given a lack of objective improvement measures, variable participant scheduling, inconsistent participant adherence, and the need to alter existing participant sleep habits for study purposes. In a study assessing the impact of noise-masking earbuds on self-reported sleepiness, sleep quality, stress level, and post-overnight shift alertness in emergency medicine resident physicians, we observed low rates of participant accrual and retention despite participants being allowed to keep the study technology on completion of the investigation. In this conceptual piece we briefly describe our findings, review conceptual challenges we faced studying this wellness intervention in our live population, and present solutions to improve 
future studies of digital sleep interventions in healthcare shift workers.

\section{Methods}

\subsection{Study design and setting}

This was a prospective, single-subject design study performed at an urban, academic, medical center which hosts residency training programs for multiple specialties including a four-year Emergency Medicine (EM) training program. This work was approved by the local Institutional Review Board.

\subsection{Participant Recruitment}

EM resident physicians working full-time in the emergency department (ED) during the study period were recruited for participation. Inclusion criteria required participants to be hearing-capable, and to own a smart phone with text messaging functionality and internet connectivity for electronic survey participation. Participants scheduled to work outside of the ED or who were off-service for any part of the study period were excluded. Participants were recruited via email to the residency listserv and through text message to a resident-specific group messaging application delivered to their personal devices. All participants provided informed consent prior to study participation.

Within this residency training program, each resident works between 18-21 shifts in a rotating schedule which includes 5-7 overnight shifts in a 28-day period. Shift hours include day shifts (approximately 7 am-5 pm), swing shifts (roughly 3 pm-2 am), and overnight shifts (roughly 11 pm-8 am).

\subsection{Interventions}

Prior to the study, participants completed an electronic entrance survey which included questions about their baseline sleep habits, sleep aid use, selfreported sleep quality, daily sleepiness, and daily tension over the prior 28-day period (Appendix 1). The study period started on the first day of a new schedule block when all recruited participants were scheduled to work in the ED over the entirety of the 28-day study period. Over the first 14 days (the control period), participants were instructed to continue using their baseline sleep habits. Beginning on study day 15 , each participant was provided with a pair of Bose SleepBuds $^{\mathrm{TM}}$ (Bose Corporation, Framingham, MA) and advised as to device functionality. Participants were instructed to use the earbuds for their sleep episodes over the next 14 days as needed (the intervention period). During the intervention, earbuds were used at the discretion of each participant, and participants were allowed to continue using other baseline sleep aids in addition to the earbuds if they so choose. At the end of the study period, participants completed an electronic exit survey asking about sleep aid use and earbud function over the prior 28-day period (Appendix 2). Participants were allowed to keep their earbuds after the study period for their own personal use.

Sleepbuds $^{\mathrm{TM}}$ (hereby referred to as earbuds) are wireless noise-masking buds designed to be worn in each ear all night (Figure 1A and B). They mask ambient noises by playing one of several sound tracks selectable from the Bose application which is accessed on the participant's cellular smartphone (Figure 1C). While there is an element of passive noise attenuation that occurs from simply wearing the earbuds (similar in functionality to earplugs), noise-masking does not take effect unless the device is connected to the application and is playing one of the selectable soundtracks. Battery life for the earbuds alone is estimated to be 16 hours of continuous play from one full charge.

A
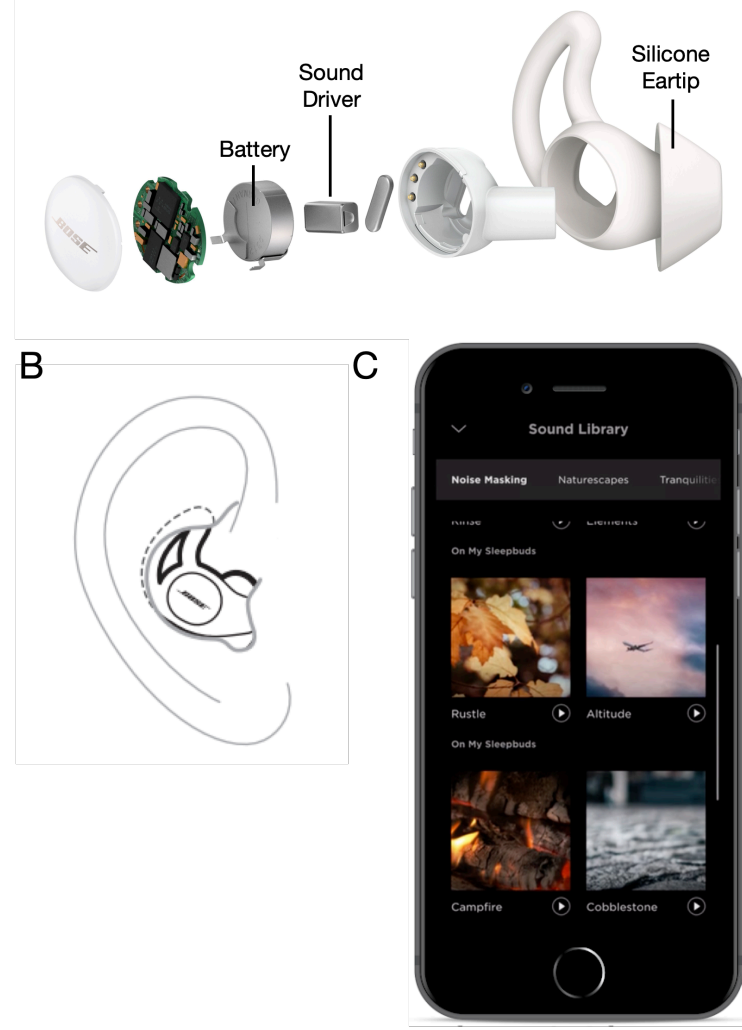

Figure 1. Earbud product used in this study. A. A silicone eartip is combined with a sound driver and battery to provide noise-masking sounds throughout the sleep period. B. The device is intended to fit comfortably in users' ears for an extended period. $C$. The device connects 
wirelessly to an application on users' phones where various soothing soundtracks are selected and played to activate the noise-masking technology. Adapted from www.bose.com with permission.

\subsection{Measurements}

2.4.1 Daily surveys. Participants received an automated text message containing a link to a daily survey on each day of the 28-day study period. Surveys asked participants to rate the quality of their last sleep episode, current daytime sleepiness, and current level of tension. All measures were reported on 8-point Likert scales of 0 (extremely bad sleep quality, not sleepy at all, and not tense at all) to 7 (extremely good sleep quality, extremely sleepy, and extremely tense). Beginning on day 15 , daily surveys also included a question about whether earbuds were used during their last sleep episode.

2.4.2. Psychomotor vigilance testing. Postovernight shifts, each participant was approached by a study research assistant to complete a 3-minute psychomotor vigilance test (PVT). This test conducted on portable tablets required participants to rapidly tap a circle as it appeared on a tablet screen, and reaction time for each tap was recorded.[13,14] Participants were able to decline participation in a daily PVT if clinical demands or other activities required their immediate attention.

2.4.3. 6-month follow-up. Participants were sent an electronic survey six months after study completion asking about sleep aid use since study completion, including earbud use. For participants still using earbuds, additional questions about when and how they most commonly used the device since the study period were included.

\subsection{Data Management and Statistical Analyses}

Study data were collected and managed using REDCap electronic data capture system sponsored and hosted at our institution. Data were de-identified prior to analysis, and analyzed using Stata 16 (StataCorp). Frequencies and percentages were used to summarize binary and categorical variables. Mean with standard deviation or median and interquartile range were used to summarize time variables and Likert scale data.

Changes in quality of last sleep episode, current daytime sleepiness, and current level of tension in control and intervention periods were assessed using a linear mixed effects regression model with a participantspecific random intercept. In the post-implementation period, we only included data collected on days when participants reported using the earbuds. For PVT data, each participants' mean reaction times pre- and postintervention period were directly compared. 6-month follow up responses were qualitatively assessed.

\section{Results}

\subsection{Study participant characteristics}

38 of the 58 invited participants enrolled in the study. Of these, six participants did not complete any daily surveys, and another 6 participants never used the earbuds thus they were excluded from final analysis. The remaining 26 participants completed a total of 655 $(89.9 \%)$ daily sleep surveys of a possible 728 surveys. Surveys taken on post-intervention days when participants reported not using earbuds were excluded, resulting in a total of 501 daily surveys being included in the final analysis (Figure 2). Of these, 344 (68.7\%) of responses were collected from the control period and $157(31.3 \%)$ of responses were collected from the intervention period. Participants included in final analysis represented resident physicians from all 4 postgraduate years. $27 \%$ of participants included in final analysis were female, and ages ranged from 25 to 35 years (Table 1).

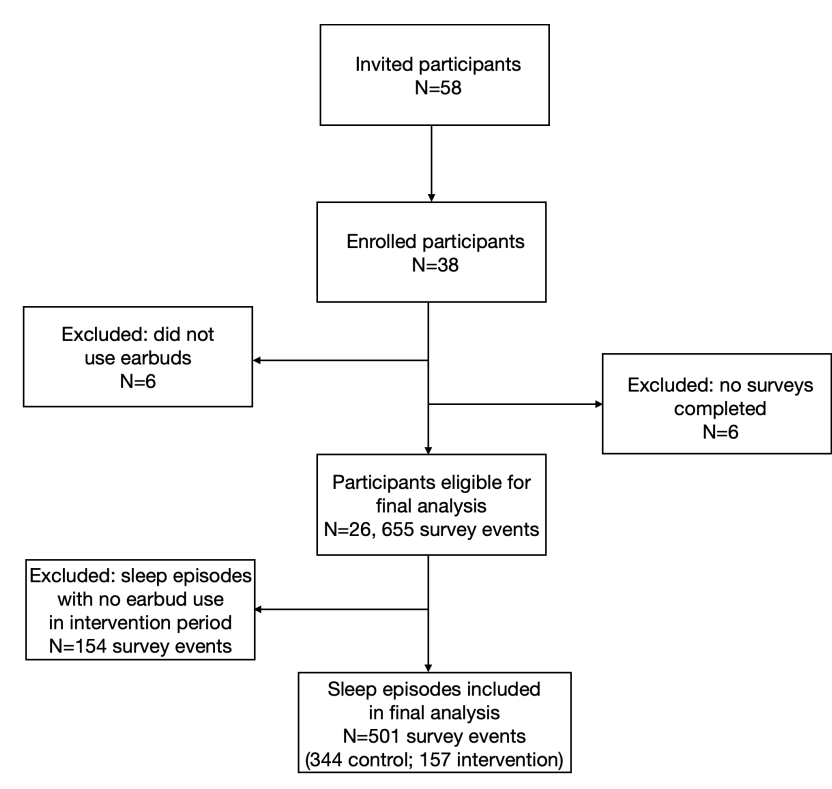

Figure 2. Study design flow chart. 
Table 1. Participant demographics.

\begin{tabular}{|c|c|}
\hline Variable & $\begin{array}{c}\text { Participants } \\
(\text { Number, } \% ; N=26)\end{array}$ \\
\hline $\begin{array}{l}\text { Sex } \\
\quad \text { Male } \\
\text { Female }\end{array}$ & $\begin{array}{c}19(73.1 \%) \\
7(26.9 \%)\end{array}$ \\
\hline $\begin{array}{c}\text { Age (years) } \\
25-30 \\
30-35\end{array}$ & $\begin{array}{l}12(46.1 \%) \\
14(53.9 \%)\end{array}$ \\
\hline $\begin{array}{l}\text { Training year } \\
\text { PGY1 } \\
\text { PGY2 } \\
\text { PGY3 } \\
\text { PGY4 }\end{array}$ & $\begin{array}{l}5(19.2 \%) \\
9(34.6 \%) \\
7(26.9 \%) \\
5(19.2 \%)\end{array}$ \\
\hline $\begin{array}{l}\text { Baseline average sleep/tension } \\
\text { measures } \\
\text { Sleep quality } \\
\text { Sleepiness } \\
\text { Tension } \\
\end{array}$ & $\begin{array}{c}5[4,6] \\
4.5[4,5] \\
5[3,5]\end{array}$ \\
\hline $\begin{array}{l}\text { Baseline sleep aid use } \\
\text { Blackout Curtains } \\
\text { Eye mask } \\
\text { Earplugs } \\
\text { Weighted blanket } \\
\text { White noise } \\
\text { Pharmacological sleep aid } \\
\text { Other } \\
\text { None of the above }\end{array}$ & $\begin{array}{c}16(61.5 \%) \\
8(30.8 \%) \\
6(23.1 \%) \\
1(3.8 \%) \\
9(34.6 \%) \\
9(34.6 \%) \\
1(3.8 \%) \\
2(7.7 \%)\end{array}$ \\
\hline
\end{tabular}

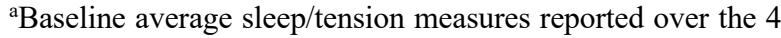
weeks prior to study participation, reported as median [IQR $\}$.

\subsection{Earbud use}

Frequency of earbud use varied highly among participants who reported using earbuds at least once during the study period. Participants most commonly used earbuds either $0-10 \%$ or $90-100 \%$ of sleep periods over the 14-day study period (Figure 3A). Of the 14 days when earbuds were available, participants used earbuds for a median of 5 days (IQR [2,9]). The lowest earbud use occurred on day one of the intervention, with 6 out of 26 participants reporting use, trending up until day 4 when 15 out of 26 participants reported using earbuds and remaining generally stable with a slight downward trend in use towards the end of the study (Figure 3B). Participants who reported using earbuds for 7 or more out of the 14 days did not have significantly different baseline pre-study 4-week sleep scores than participants who reported using earbuds between 1 to 6 days (mean score 5.2 vs $4.8, P=0.34$ ).
A

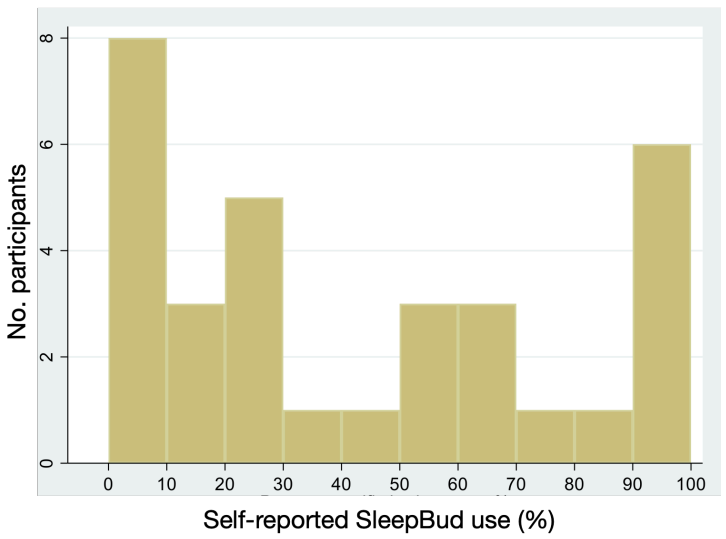

B

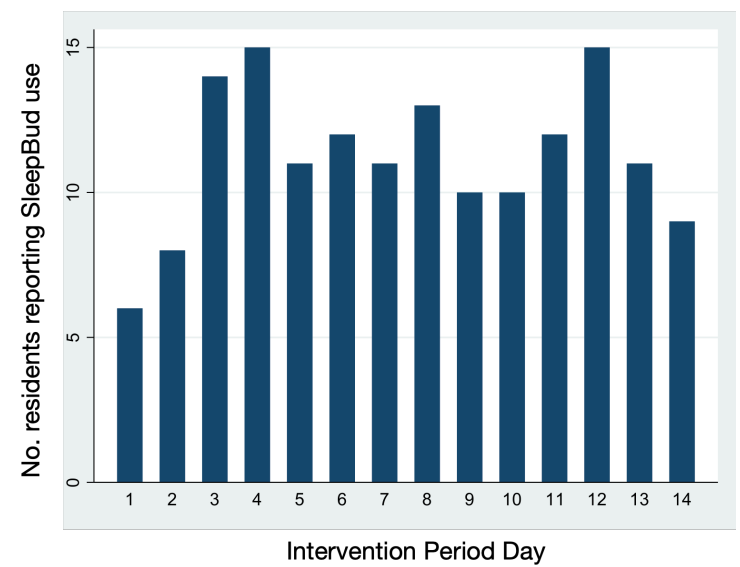

Figure 3. Self-reported earbud use over the intervention period. A. Percentage of sleep periods during which earbuds were used by participants throughout the total intervention period. B. Number of participants reporting earbuds use per night over the course of the intervention period.

\subsection{Sleep quality, daily sleepiness, and tension}

Participants' self-reported last sleep episode quality, daily sleepiness, and daily tension had improving trends in the post-intervention period (Figures 4A-C). On days when residents used earbuds, on Likert scales of 0 to 7, previous nights' sleep quality increased by 0.5 points $(P<.0001,95 \%$ CI $0.23-0.80)$, daily sleepiness decreased by 0.6 points $(P<.0001,95 \%$ CI -0.90 to -0.34$)$, and total daily tension decreased by 0.5 points $(P<.0001,95 \%$ CI -0.81 to -0.32$)$ using linear mixed regression models. In the subset of participants who reported below-median sleep scores preintervention, the beneficial effects of using earbuds were amplified. 
A

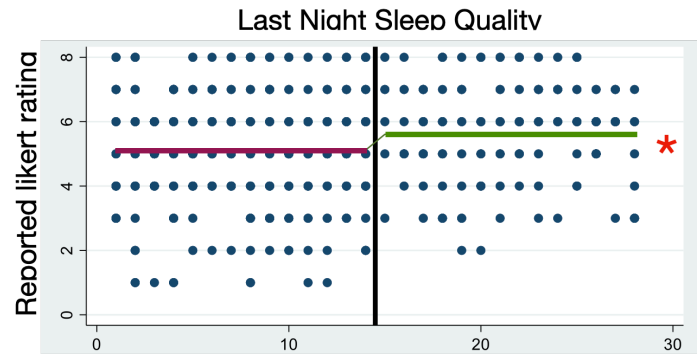

B

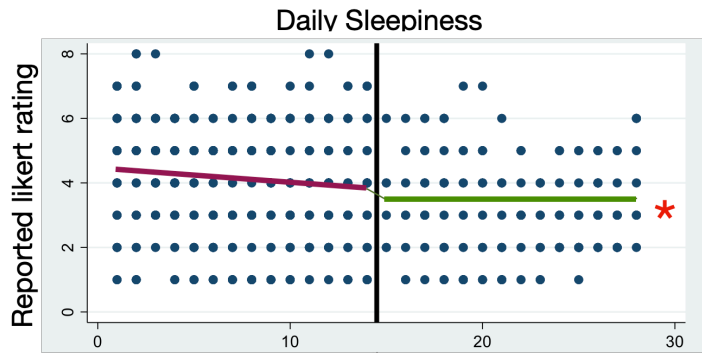

C

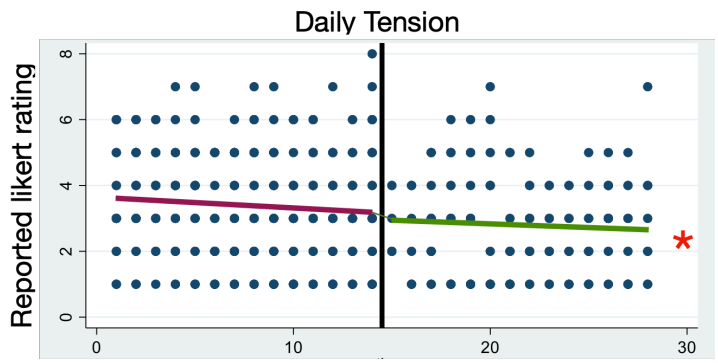

Daily rating

Pre-Bud trend

Post-Bud trend

Figure 4. Regression analysis of self-reported last night sleep quality (A), daily sleepiness (B), and daily tension (C) in pre- and post-earbud use periods. $\mathrm{X}$-axis represents the study day with the midline representing the transtion from the control to the experimental periods. Red stars represent significance from pre- to post-earbud ratings.

\subsection{Post-night shift alertness and reaction time}

In total seven post-overnight shift residents completed a total of 12 PVT tests during the intervention period. Six participants completed PVTs were linked to participants who reported no earbud use thus were excluded from final analysis. Ultimately three participants both used the earbuds and completed PVTs in both the control and intervention periods. Data from these participants comparing pre- and post-earbuds mean reaction time showed no statistical differences. However, this study was underpowered to detect an effect of earbud use on PVT.

\subsection{6-month follow up}

We received responses from $12(46 \%)$ participants. Of the 12 respondents, 5 reported ongoing earbud use; all of whom reported use on less than $25 \%$ of all sleep episodes since study completion. Participants cited limited sound options, uncomfortable fit, and forgetting to use the device as barriers to further use. There were no notable differences in self-reported baseline sleep aid use and 6-month follow up sleep aid use.

\section{Discussion}

Here we discuss our experience studying the realworld uptake and effect of a digital health intervention on sleep in a population of healthcare shift workers. While we were able to detect small yet significant improvements in self-reported last nights' sleep quality, daily sleepiness, and daily tension, we observed relatively low rates of study enrollment and participant adherence which impacted the robustness of our findings. Further, our objective measures of sleep were improved by variable participant shift scheduling combined with the relatively brief duration of our study period. Implementing wellness interventions aimed at improving sleep among vulnerable populations such as healthcare shift workers is an important goal. In order to initiate robust prospective studies evaluating possible future interventions, methods must balance targeting study enrollment, participant adherence to study protocols, and optimizing intervention uptake long term.

\subsection{Optimizing study enrollment}

Approximately $65 \%$ of potential participants who were invited actually enrolled in this study. We attempted to improve enrollment by offering the earbuds free of charge to keep for personal use after the study period. Although we did not quantify these responses, eligible individuals indicated that the technology was highly valued by our target population. Despite their reported desirability, the technology did not appear to trigger the anticipated interest among eligible residents. Further, while offering the device free-of-charge may have recruited participants who were motivated to enroll for reasons other than their intent for meaningful participation, this may have created a self-selection bias. To address this problem in future works, we recommend establishing a threshold of study protocol adherence rate for participants to qualify to be allowed to keep the devices on study completion.

Given that adequate sleep among shift workers is highly desired yet often quite tenuous, one possible explanation for low study enrollment is that participants 
are reluctant to engage in interventions that have even the theoretical possibility of disrupting sleep. Concerns about the potential for uncomfortable fit, or the sounds tracks being disruptive to sleep were cited among eligible residents who chose not to enroll. We tried to mitigate this risk by designing the study so that participants were only asked to use earbuds as needed, however this resulted in overall poor study adherence. Interestingly, a larger proportion of males enrolled in the study compared to females despite the fact that the group initially solicited was roughly even in terms of sex distribution. This may suggest that technology-based interventions for sleep are more attractive to males than females. Ultimately, initiating focus groups discussing product functionality, desirability across diverse groups of users, and potential concerns regarding product use could have allowed for us to integrate strategies for both enhanced participant enrollment and potentially adherence to study protocols.

\subsection{Optimizing study protocol adherence}

Among enrolled participants, we observed highly variable rates of protocol adherence both in terms of earbud use and survey responses over the study duration. Most common patterns reflected earbud use in a nearly bimodal distribution with median earbud use occurring for only 5 out of the possible 14 days. Concerns about uncomfortable device fit, difficulty with fitting the device in to their existing sleep routine, and simply forgetting to use the device were cited as barriers to adherence. For future studies, we recommend first performing a brief pilot study in which participants use the device for a short period of time and report back challenges impacting device use. This pilot data would give opportunity for identified challenges to be addressed in the protocol to ideally improve intervention adherence.

Many healthcare shift workers develop habits and behaviors for addressing sleep disturbances early in their careers. To address participants forgetting to use the intervention or reporting that the intervention did not fit in to their established sleep habits, initiating interventions early in training before habits are solidified may improve intervention uptake. Performing a more longitudinal study spanning several months as opposed to a single month may also allow for the intervention to be regularly established into nightly sleep patterns and thus improve adherence over time. Further, we could also offer regular reminders to use the intervention potentially in the form of automated text message delivered to participant smartphones. This gets somewhat complicated when studying a live population however as we would ideally time the reminder shortly before participants initiate their sleep period which means we would have to correlate the timing of the text message with each participants' shift schedule. Similarly, reminders to complete study surveys could be delivered via text message at regular intervals to improve survey response rate.

\subsection{Maximizing measures of objective outcomes}

Self-reporting subjective outcome data is a common practice in sleep intervention studies performed in real-world populations.[15-17] Here, outcome measures of daily sleepiness, last nights' sleep quality, and daily tension were manually reported by participants via brief daily surveys delivered by text message. Even among participants who used the earbuds in the study, consistency with filling out daily surveys was lacking. Health care workers are often bombarded with surveys and requests for task completion, thus asking them to remember to manually complete daily surveys may not be realistic. Data from the surveys also relied on self-reporting from participants, which is inherently limited and potentially biased. For sleep interventions such as this, data collection that is automated and minimizes user interaction may be both more ideal and accurate.

In the present study, we used PVT as a means of objective data collection on the impact of sleep on alertness. PVT is a well-established and even goldstandard measure for identifying variations in alertness and performance related to sleep.[14] However, as occurred in our study, obtaining PVT data requires participant interaction with study personnel which may not be practical based on rotating schedules or participant availability. Approximately $1 / 3$ of resident shifts are overnight, and night shifts tend to be clustered and are not evenly distributed over a 28-day period. Thus, typical resident schedules are somewhat suboptimal for a single-subject design where subjects serve as their own controls across only 28 -days.

Future solutions to optimizing PVT data may include recruiting study participants such as nocturnists who are physicians who work a higher proportion and more consistent distribution of night shifts, or other groups of shift workers who work exclusively overnight. Increasing study population size or extending the study period by multiple months would likely also enhance our data points and improve study power.

In addition to PVT data, collecting automated or passively collected, objective measures of sleep quality without the need for participant interaction would improve studies assessing sleep interventions. Several options such as commercial applications deployed on participant's smartphones or wearable devices measuring markers of sleep currently exist. Options such as wearable acceleromaters, or fitness trackers, or 
even under-mattress sensors which could potentially offer objective data on sleep outcomes and should be employed in future studies.[18-21]

\subsection{Limitations - additional considerations}

This was a single-arm, case-control study with a small sample size, and limited 28-day study period. All of these factors affected the analytical power of our study. Though this work had the benefit of prospective data collection, this work can be used as pilot to inform future randomized controlled trials comparing earbud use to matched controls. Our work focused on resident physicians as representatives of the healthcare shift worker population. This likely introduces bias in assessing a digital intervention as this population tends to be fairly young in age and may be more willing to uptake new technology compared to the shift worker population as a whole. Ideally assessing the effect of a digital intervention in as varied a population as possible would be ideal.

\subsection{Conclusions}

Sleep loss is a public health crisis that disproportionately affects shift workers and, in particular healthcare, workers. This not only impacts the mental and physical health of front-line workers, but can also negatively impact patient care and outcomes. Despite this, institutional wellness initiatives aimed at improving sleep are lacking. Given the global scale of this wellness and health concern, optimizing studies assessing low-risk, feasible, digital interventions are key. Here, we discuss challenges associated with such studies, and propose several solutions to optimize the overall feasibility of evaluating digital health interventions in real-world populations of healthcare shift workers. A summary of our recommendations can be found in Table 2. Interventions such as the earbuds studied here have the potential to not only improve employee health and wellness, but if implemented correctly these benefits may also be able to be applied to improve the sleep and wellbeing of patients in healthcare settings, or shift workers outside of healthcare as well.
Table 2. Recommendations for optimizing digital sleep intervention studies in real-time.

\begin{tabular}{|c|c|}
\hline Study Element & $\begin{array}{c}\text { Recommended Optimization } \\
\text { Strategies }\end{array}$ \\
\hline $\begin{array}{l}\text { Participant } \\
\text { recruitment }\end{array}$ & - $\quad$ Pre-study focus groups \\
\hline $\begin{array}{l}\text { Intervention } \\
\text { adherence }\end{array}$ & $\begin{array}{l}\text { - } \quad \text { Pilot study } \\
\text { - } \quad \text { Daily automated } \\
\text { reminders }\end{array}$ \\
\hline $\begin{array}{l}\text { Study protocol } \\
\text { adherence }\end{array}$ & $\begin{array}{l}\text { - Daily automated } \\
\text { reminders } \\
\text { - Minimum required } \\
\text { participation to retain } \\
\text { device post-study }\end{array}$ \\
\hline $\begin{array}{l}\text { Robust outcome } \\
\text { measures }\end{array}$ & $\begin{array}{l}\text { - } \quad \text { Larger study size } \\
\text { - } \quad \text { Longitudinal study } \\
\text { - } \quad \text { Recruit from } \\
\text { populations with more } \\
\text { uniform shift schedules } \\
\text { - Data collection from } \\
\text { automated measures such } \\
\text { as wearable devices, } \\
\text { applications or biosensors } \\
\text { - Prioritize objective } \\
\text { data collection }\end{array}$ \\
\hline
\end{tabular}

\section{References}

[1] Liu Y, Wheaton AG, Chapman DP, et al. Prevalence of Healthy Sleep Duration among Adults - United States, 2014. CDC MMWR. Feb 19, 2016: 65(6); 137-141.

[2] Shockey TM and Wheaton AG. Short Sleep Duration by Occupation Group- 29 states, 2013-2014. CDC MMWR. March 3, 2017: 66(8); 207-213.'

[3] Di Milia L, Waage S, Pallesen S, Bjorvatn B. Shift work disorder in a random population sample--prevalence and comorbidities. PLoS One. 2013;8(1):e55306. doi: 10.1371/journal.pone.0055306. Epub 2013 Jan 25. PMID: 23372847

[4] Ganesan S, Magee M, Stone JE, Mulhall MD, Collins A, Howard ME, Lockley SW, Rajaratnam SMW, Sletten TL. The Impact of Shift Work on Sleep, Alertness and Performance in Healthcare Workers. Sci Rep. 2019 Mar 15;9(1):4635. doi: 10.1038/s41598-019-40914-x. PMID: 30874565

[5] Kerkhof GA. Shift work and sleep disorder comorbidity tend to go hand in hand. Chronobiol Int. 2018 Feb;35(2):219-228. doi: 10.1080/07420528.2017.1392552. Epub 2017 Nov 20. PMID: 29157012

[6] Wickwire EM, Geiger-Brown J, Scharf SM, Drake CL. Shift Work and Shift Work Sleep Disorder: Clinical and Organizational Perspectives. Chest. 2017 May;151(5):1156-1172. doi: 10.1016/j.chest.2016.12.007. Epub 2016 Dec 21. PMID: 28012806 
[7] Bureau of Labor Statistics. (2019, September). Job Flexibilities and Work Schedules Summary. (USDL-191691). Retrieved September 29, 2020, from https://www.bls.gov/news.release/flex2.nr0.html.

[8] Basner M, Dinges DF, Shea JA, Small DS, Zhu J, Norton L, Ecker AJ, Novak C, Bellini LM, Volpp KG. Sleep and Alertness in Medical Interns and Residents: An Observational Study on the Role of Extended Shifts. Sleep. 2017 Apr 1;40(4):zsx027. doi: 10.1093/sleep/zsx027. PMID: 28329124

[9] Arzalier-Daret S, Buléon C, Bocca ML, Denise P, Gérard JL, Hanouz JL. Effect of sleep deprivation after a night shift duty on simulated crisis management by residents in anaesthesia. A randomized crossover study. Anaesth Crit Care Pain Med. 2018 Apr;37(2):161-166. doi: 10.1016/j.accpm.2017.05.010. Epub 2017 Sep 4. PMID: 28882740.

[10] Kalmbach DA, Arnedt JT, Song PX, Guille C, Sen S. Sleep Disturbance and Short Sleep as Risk Factors for Depression and Perceived Medical Errors in First-Year Residents. Sleep. 2017 Mar 1;40(3):zsw073. doi: 10.1093/sleep/zsw073. PMID: 28369654

[11] Trockel MT, Menon NK, Rowe SG, Stewart MT, Smith R, Lu M, Kim PK, Quinn MA, Lawrence E, Marchalik D, Farley H, Normand P, Felder M, Dudley JC, Shanafelt TD. Assessment of Physician Sleep and Wellness, Burnout, and Clinically Significant Medical Errors. JAMA Netw Open. 2020 Dec 1;3(12):e2028111. doi: 10.1001/jamanetworkopen.2020.28111. PMID: 33284339

[12] Robbins R, Weaver MD, Quan SF, Rosenberg E, Barger LK, Czeisler CA, Grandner MA. (2020). Employee Sleep Enhancement and Fatigue Reduction Programs: Analysis of the 2017 CDC Workplace Health in America Poll. American Journal of Health Promotion, 890117120969.

[13] Basner M, Dinges DF. Maximizing sensitivity of the psychomotor vigilance test (PVT) to sleep loss. Sleep. 2011;34(5):581-591. Published 2011 May 1. doi:10.1093/sleep/34.5.581

[14] Ferris M, Bowles KA, Bray M, Bosley E, Rajaratnam SMW, Wolkow AP. The impact of shift work schedules on PVT performance in naturalistic settings: a systematic review. Int Arch Occup Environ Health. 2021 Oct;94(7):1475-1494. doi: 10.1007/s00420-02101668-0.

[15] Robbins R, Jackson CL, Underwood P, Vieira D, JeanLouis G, Buxton OM. Employee Sleep and Workplace Health Promotion: A Systematic Review. Am J Health Promot. 2019 Sep;33(7):1009-1019. doi: 10.1177/0890117119841407.

[16] Robbins R, Underwood P, Jackson CL, Jean-Louis G, Madhavaram S, Kuriakose S, Vieira D, Buxton OM. A Systematic Review of Workplace-Based Employee Health Interventions and Their Impact on Sleep Duration Among Shift Workers. Workplace Health Saf. 2021 Jul 20:21650799211020961. doi: $10.1177 / 21650799211020961$.

[17] Sletten TL, Raman B, Magee M, Ferguson SA, Kennaway DJ, Grunstein RR, Lockley SW, Rajaratnam SMW. A Blue-Enriched, Increased Intensity Light
Intervention to Improve Alertness and Performance in Rotating Night Shift Workers in an Operational Setting. Nat Sci Sleep. 2021 May 24;13:647-657. doi: $10.2147 /$ NSS.S287097.

[18] Sundararajan K, Georgievska S, Te Lindert BHW, Gehrman PR, Ramautar J, Mazzotti DR, Sabia S, Weedon MN, van Someren EJW, Ridder L, Wang J, van Hees VT. Sleep classification from wrist-worn accelerometer data using random forests. Sci Rep. 2021 Jan 8;11(1):24. doi: 10.1038/s41598-020-79217-x.

[19] Nagatomo K, Masuyama T, Iizuka Y, Makino J, Shiotsuka J, Sanui M. Validity of an under-mattress sensor for objective sleep measurement in critically ill patients: a prospective observational study. J Intensive Care. 2020 Feb 11;8:16. doi: 10.1186/s40560-020-0433$\mathrm{x}$.

[20] McDonald L, Mehmud F, Ramagopalan SV. Sleep and BMI: Do (Fitbit) bands aid? F1000Res. 2018 Apr 27;7:511. doi: 10.12688/f1000research.14774.2.

[21] de Zambotti M, Goldstone A, Claudatos S, Colrain IM, Baker FC. A validation study of Fitbit Charge 2 ${ }^{\mathrm{TM}}$ compared with polysomnography in adults. Chronobiol Int. 2018 Apr;35(4):465-476. doi: 10.1080/07420528.2017.1413578. 


\section{Appendix 1. A) Entrance survey and B) exit survey completed by all participants.}

A. SleepBuds ${ }^{\mathrm{TM}}$ - Enrollment Survey

Demographics:

1. Name

2. Phone number to receive daily text messages

3. Date of birth

4. Gender

5. Residency year

6. Last rotation

7. Current rotation

Current Sleep Aids:

8. Current use of sleep aids:
a. Blackout Curtains
b. Eye mask
c. Earplugs
d. Weighted blanket
e. White noise
f. Pharmacological sleep aid
g. Other (describe below)
h. None of the above

Current Sleep Quality:

9. How would you evaluate your average sleep over the last 4 weeks? (Likert 0-7, extremely bad to extremely good)

10. How sleepy have you felt on an average day over the last 4 weeks? (Likert 0-7, not sleepy at all to extremely sleepy)

11. How tense do you feel on an average day over the last 4 weeks? (Likert scale 0-7, not tense at all to extremely tense)

\section{B. SleepBuds ${ }^{\mathrm{TM}}$ - Exit Survey}

1. Name

2. Do you use any of the following as sleep aids? (select all that apply)
i. Blackout Curtains
j. Eye mask
k. Earplugs
1. Weight blanket
m. White noise machine
n. Pharmacologic sleep aid
o. None of the above

3. Have you decreased your use of these sleep aids over the last 4 weeks? (Likert 0-7, significant decreased to no change)

Current Sleep Quality:

4. How would you evaluate your last sleep period? (Likert 0-7, extremely bad to extremely good)
5. How sleepy do you feel today? (Likert 0-7, not sleepy at all to extremely sleepy)

6. How tense do you feel today? (Likert scale 07 , not tense at all to extremely tense)

7. Did you use the SleepBuds ${ }^{\mathrm{TM}}$ during your last sleep period? (yes, no)

8. What was the most recent shift period you worked in the last 24 hours? (Day shift, Afternoon/Evening shift, Overnight shift, Did not work in the last 24 hours) 